\title{
Estudo sobre a Adoção de Práticas de Contabilidade Gerencial em Empresas Hoteleiras de Florianópolis, SC, Brasil
}

\author{
Rogério João Lunkes ${ }^{a}$ \\ Carla Helen Costab \\ Daiane Antonini Bortoluzzic \\ Fabricia Silva da Rosad
}

\section{Resumo}

O objetivo deste estudo é analisar a adoção de práticas de contabilidade gerencial em empresas hoteleiras. Para atingir esse objetivo, um questionário foi aplicado a 15 hotéis da grande Florianópolis, SC, Brasil, filiados à Associação Brasileira da Indústria de Hotéis (ABIH), no período de abril de 2014 a maio de 2016. Com essa limitação, os resultados servem para o escopo específico e mostram que a contabilidade gerencial é adotada em grande parte das empresas hoteleiras pesquisadas. Dos resultados, pode-se destacar o uso dos métodos de custeio por absorção e de custeio padrão, a utilização do orçamento para planejamento anual e controle de custos, medidas de rentabilidade e análise de rentabilidade do produto e de clientes, e análise dos pontos fortes e fracos e da posição dos concorrentes. Isso mostra que geralmente os hotéis adotam práticas tradicionais, de primeiro e segundo estágio. Entre as possíveis mudanças apontadas pelos gestores hoteleiros para os próximos três anos está a utilização de medidas não financeiras relacionadas à inovação e à gestão baseada em atividades.

Palavras-chave: Contabilidade gerencial; Estágios; Práticas; Hotel; Florianópolis.

\section{Abstract \\ Study on the adoption of management accounting practices in hotels in Florianópolis, SC, Brazil}

This study aims to analyze the adoption of management accounting practices in hotels. To reach this objective, a questionnaire was applied to 15 hotels in the metropolitan region of Florianópolis, Santa Catarina (Brazil), affiliated to ABIH (Brazilian Hotel Industry Association), from April 2014 to May 2016. Through this accounting, the results serve as specific scope and show management accounting is adopted in most of the hotels. Among those results, it can be highlighted the use of absorption costing and standard costing methods; budget use for annual planning and cost control; measures and analysis

a. Possui pós-doutorado em Contabilidade pela Universidade de Valência, Valência, Valência, Espanha. Professor do curso de Ciências Contábeis e do Programa de Pós-Graduação em Contabilidade e Administração da Universidade Federal de Santa Catarina, Florianópolis, Santa Catarina, Brasil. E-mail: rogerio.lunkes@ufsc.br

b. Bacharel em Ciências Contábeis pela Universidade Federal de Santa Catarina, Florianópolis, Santa Catarina, Brasil. E-mail: carlacnec@hotmail.com

c. Doutora em Administração pela Universidade Federal de Santa Catarina, Florianópolis, Santa Catarina, Brasil. E-mail: daianeantonini@gmail.com

d. Possui pós-doutorado em Contabilidade pela Universidade Federal de Santa Catarina, Florianópolis, Santa Catarina, Brasil. Professora do curso de Ciências Contábeis e do Programa de Pós-Graduação em Contabilidade na mesma instituição. E-mail: fabriciasrosa@hotmail.com 
of product and customers profitability; and strengths and weaknesses and competitors position analysis. This shows that hotels usually adopt first and second stage traditional practices. The use of non-financial measures related to innovation and Activity-Based Management are among the possible changes pointed by hotel managers for the next three years.

Keywords: Management accounting; Internships; Practices; Hotel; Florianópolis.

\section{Resumen} Estudio sobre la adopción de prácticas de contabilidad gerencial en empresas
hoteleras de Florianópolis, SC, Brasil

El objetivo del estudio es analizar la adopción de prácticas de contabilidad gerencial en empresas hoteleras. Para alcanzar ese objetivo, se aplicó un cuestionario a 15 hoteles de la gran Florianópolis-Santa Catarina (Brasil), afiliadas a la ABIH (Asociación Brasileña de la Industria de Hoteles), entre abril de 2014 y mayo de 2016. Los resultados muestran que la contabilidad gerencial es adoptada en gran parte de las empresas hoteleras, siendo el destaque para: el uso de los métodos de costeo por absorción y de costeo estándar; la utilización del presupuesto para planificación anual y control de costos; medidas de rentabilidad y análisis de rentabilidad del producto y de clientes; $\mathrm{y}$ análisis de los puntos fuertes y débiles y posición de los competidores. Esto demuestra que generalmente los hoteles adoptan prácticas tradicionales, de primera y segunda etapa. Entre los posibles cambios, en los próximos tres años, apuntados por los gestores hoteleros está la utilización de medidas no financieras relacionadas con la innovación y la Gestión Basada en Actividades.

Palabras clave: Contabilidad gerencial; Pasantías; Prácticas; Hotel; Florianópolis.

\section{INTRODUÇÃO}

As empresas hoteleiras apresentam um conjunto de características que as tornam praticamente únicas. Aspectos como sazonalidade, volatilidade, perecibilidade, diversidade, trabalho intensivo, tempo reduzido na prestação do serviço, investimento elevado em ativos fixos e custos fixos elevados ajudam a explicar essa singularidade (Lima Santos et al., 2016).

Essas características agregam complexidade à gestão hoteleira, o que demanda configurações e práticas de gestão personalizadas. A contabilidade gerencial (CG) proporciona um suporte de informações financeiras e não financeiras de apoio ao processo de tomada de decisão. Ela visa fornecer aos gestores informações necessárias para o planejamento, o controle e a tomada de decisão (Jiambalvo, 2000). Para realizar o processo de gestão, a empresa hoteleira pode utilizar diferentes práticas relacionadas a custos, orçamento, mensuração e avaliação de desempenho.

A adoção dessas práticas, que podem ser classificadas em quatro estágios evolutivos (Abdel-Kader \& Luther, 2006), proporciona diferentes níveis de informação. Essas práticas de CG são classificadas por método de custeio, orçamento, avaliação de desempenho, tomada de decisão e análise estratégica. A literatura vem destacando a utilização de práticas modernas de CG, como o orçamento de base zero, o orçamento flexível e o orçamento baseado em atividades (Codesso, Lunkes, \& Suave, 2013; Pavlatos \& Paggios, 2009; Souza \& Lunkes, 2015; Steed \& Gu, 2009; Uyar \& Bilgin, 2011); medidas não financeiras; 
e ferramentas como o valor econômico agregado (EVA) e o balanced scorecard (Kaplan \& Norton, 1997; Souza, Souza, Petri, \& Lunkes, 2015). Essas práticas podem melhorar sensivelmente as informações utilizadas no processo de tomada de decisão dos gestores hoteleiros.

A literatura, no entanto, tem apontado uma baixa utilização de práticas modernas de CG (Bain \& Company, 2013; Castro, De Souza, Rockenbach, \& Lunkes, 2012; Deboleto, Hall, Dutra, \& Silva, 2013) nas empresas hoteleiras (Atkinson \& Brown, 2001; Leitão \& Silva, 2009; Lima, Egito, \& Silva, 2004; Uyar \& Bilgin, 2011), o que pode comprometer a manutenção do empreendimento no mercado diante de organizações mais competitivas (Atkinson \& Brown, 2001; Lima, Egito, \& Silva, 2004).

Para compreender essa problemática, elaborou-se a seguinte pergunta de pesquisa: quais são as práticas de CG adotadas pelas empresas hoteleiras? Para respondê-la, o objetivo deste estudo é analisar a adoção dessas práticas em empresas hoteleiras. Para isso, foram pesquisadas empresas da grande Florianópolis, SC, Brasil, por meio da aplicação de questionário.

Estudos em empresas hoteleiras são importantes, pois a hotelaria representa um elo fundamental no ramo do turismo, o qual contribui diretamente no desenvolvimento da economia e na geração de empregos. Florianópolis, por exemplo, tem sua economia fortemente alicerçada no turismo, ocupando o $2^{\circ}$ lugar na recepção de turistas estrangeiros no Brasil (Gurgel, 2016).

Pesquisas sobre práticas de CG podem trazer insights importantes, pois pouco se conhece sobre sua utilização no ramo hoteleiro (Pellinen, 2003). A pesquisa da CG tem tradicionalmente enfocado práticas para empresas de grande porte. Portanto, a importância desta pesquisa consiste em identificar, por meio de questionário, a forma de gerenciamento dos hotéis.

Além desta introdução, o restante deste artigo está organizado da seguinte forma: a próxima seção analisa a literatura relevante sobre o tema e desenvolve o referencial teórico, com a descrição do conceito e dos estágios, das práticas de CG, além de estudos sobre práticas de CG em hotéis. A seção subsequente descreve a metodologia de pesquisa, a posterior apresenta os resultados da pesquisa, e a última conclui e discute suas implicações para a literatura e a prática das empresas hoteleiras.

\section{REFERENCIAL TEÓRICO}

Nesta seção, apresenta-se o conceito de CG e os estágios definidos e classificados pela Federação Internacional de Contadores (International Federation of Accountants [IFAC], 1998). Na sequência, são apresentadas as práticas de CG e, mais adiante, os estudos sobre essas práticas em empresas hoteleiras.

\section{Conceito e estágios da contabilidade gerencial}

Em 1989, a IFAC definiu CG como o processo que contribui na tomada de decisão, no desenvolvimento do planejamento e dos sistemas de gestão de desempenho, e no provimento de competências relacionadas aos relatórios financeiros e de controle. Dessa forma, a CG auxilia os gestores na formulação 
e na implementação da estratégia da organização (Institute of Management Accountants [IMA], 2008). Maximiano (2000) descreve que a CG assume a função de auxiliar os gestores a definir objetivos, conduzir a organização, controlar as atividades e avaliar o desempenho da organização. A evolução da CG com relação a seus objetivos, tarefas e parâmetros é apresentada em quatro estágios, de acordo com a IFAC (1998).

O primeiro estágio evolutivo da CG ocorre no período anterior a 1950 e é descrito como uma atividade técnica necessária para alcançar os objetivos da organização. Teve como foco principal a determinação de custos e o controle financeiro por meio do uso de orçamentos e tecnologias da contabilidade de custos (IFAC, 1998). O segundo estágio compreende o período entre 1950 e 1965 e teve como foco o fornecimento de informação, controle e planejamento empresarial por meio do uso de tecnologias como análise de decisão e contabilidade por responsabilidade (IFAC, 1998). 0 terceiro estágio, inserido entre os anos de 1965 a 1985, teve a atenção voltada para a redução de desperdício dos recursos empregados nos processos da empresa por meio da análise de processos e de tecnologias de gerenciamento de custos (IFAC, 1998). Por último, no quarto estágio, que teve início em 1985 e perdura até os dias atuais, a atenção foi transferida para a geração ou criação de valor pelo uso efetivo de recursos e de tecnologias que analisam os direcionadores de valor para o cliente e o acionista e por meio da inovação organizacional (Abdel-Kader \& Luther, 2006; IFAC, 1998).

Assim, o que diferencia os estágios 2, 3 e 4 é a mudança de foco da prestação de informações e da gestão de recursos para a redução de resíduos (estágio 3) e para a criação de valor (estágio 4). No entanto, o foco no fornecimento de informações a partir do estágio 2 não é perdido, mas reinterpretado e restruturado nos estágios 3 e 4 (IFAC, 1998). Consequentemente, a CG nas etapas 3 e 4 torna-se parte central do processo de gerenciamento, disponibilizando informações e auxiliando no processo de gestão (IFAC, 1998). O uso dos recursos (incluindo a informação) para criar valor é ponto central do processo de gestão nas organizações contemporâneas (Abdel-Kader \& Luther, 2006).

\section{Práticas de contabilidade gerencial (PCG)}

Abdel-Kader e Luther (2006) classificaram as práticas de CG (PCG) em quatro estágios, conforme o Quadro 1.

Conforme o Quadro 1, na primeira etapa evolutiva caracterizam-se a determinação dos custos e o controle financeiro. Segundo Martins (2003), os métodos de custeio foram desenvolvidos para uma melhor apropriação de custos. Assim, foram emergindo diversos métodos de custeamento, como custeio por absorção, custeio variável, custeio padrão e custeio baseado em atividades (ABC), sendo este último classificado no estágio 3 como método de redução de desperdício (Abdel-Kader \& Luther, 2006). Já o orçamento é um método que os gestores utilizam como apoio para as funções de planejamento e controle, ajudando-os a traçar objetivos (Horngren, Sundem, \& Stratton, 2004). 


\section{Quadro 1 - Práticas de contabilidade gerencial}

\section{Estágio I \\ Determinação \\ dos custos \\ e controle \\ financeiro}

Estágio II
Provisão de
informação para
planejamento
e controle
gerencial

Orçamento para controle de custos.

Medidas financeiras.

Utilização do orçamento flexível.

Base de rateio para a apropriação de custos indiretos de produção.

Avaliação de grandes investimentos no período de retorno e/ou retorno contábil.

Análises de custo/volume/lucro (ponto de equilíbrio) para os principais serviços.

Análise de lucratividade por serviços.

Orçamento para planejamento.

Separação entre custos fixos e variáveis.

Previsão em longo prazo.

Departamentalização para apropriação dos custos indiretos de produção. Avaliação de grandes investimentos de capital com base no método do fluxo de caixa descontado.

Análises baseadas em orçamento.

Medidas não financeiras relacionadas aos processos internos.

Uso (análise através de regressão ou de curvas de aprendizagem técnica).

Modelos de controle de estoques

Orçamento em longo prazo (planos estratégicos).

Orçamento baseado em atividades.

Método de custeio baseado em atividades.

Avaliação do custo da qualidade dos serviços/produtos.

Utilização de orçamento base zero.

Análise da sensibilidade do lucro em projetos de grande investimento de capital.

Utilização de análises probabilísticas ou simulações para avaliação de riscos em grandes investimentos de capital.

Medidas não financeiras relacionadas ao quadro funcional.

Análise das forças e fraquezas da concorrência.

Medidas não financeiras relacionadas aos clientes.

Análises de lucratividade por cliente.

Análise dos segmentos de serviços/produtos.

Retorno sobre os valores investidos.

Análise do ciclo de vida do produto gerado pelo serviço (desde a coleta de dados até quando a informação ainda seja útil para a tomada de decisões).

\section{Estágio IV}

Criação de valor

por meio do

uso eficaz de

Análise de valor para os acionistas.

Análise da posição competitiva.

Para avaliação de grandes investimentos de capital, aspectos não

financeiros são documentados e comunicados.

Análise da cadeia de valor.

Utilização de fluxo de caixa descontado para avaliação do custo do capital, em grandes investimentos.

Metodologia do custo-alvo

Avaliação de desempenho com base no lucro residual.

Benchmarking.

Fonte - Abdel-Kader e Luther (2006) 
Na literatura de CG e segundo a classificação de Abdel-Kader e Luther (2006), o orçamento surge como parte da informação para o planejamento e para o CG, tornando-se uma técnica essencial para planejar e controlar as atividades nas organizações (Drury, Braund, Osborne, \& Tayles, 1993), além de proporcionar diretrizes, objetivos, metas e indicadores estabelecidos pelos altos executivos (Souza \& Lunkes, 2015).

Para enfrentar os desafios do mercado competitivo, as empresas precisam desenvolver técnicas de avaliação de desempenho (Nudurupati, Bititci, Kumar, \& Chan, 2011). Nesse cenário, escolher medidas que orientam e avaliam o desempenho das empresas torna-se um dos desafios mais críticos das organizações (Ittner \& Larcker, 1998). Portanto, a avaliação de desempenho é um processo para analisar problemas complexos que envolve múltiplos critérios de decisão. Tem como base um processo para identificar e agrupar em clusters o conhecimento gerado em documentos (Aliguliyev, 2009). Considera-se, ainda, que o contexto da avaliação de desempenho utilizado para analisar processos (Balasubramanian \& Gupta, 2005) e identificar pontos fortes e fracos do contexto, melhorando a aprendizagem contínua (Chin, Lo, \& Leung, 2010), seja singular (Abernethy, Horne, Lillis, Malina, \& Selto, 2005).

Segundo Abdel-Kader e Luther (2006), a CG deve relatar informações pertinentes à avaliação de desempenho, incluindo medidas financeiras, tais como lucros, retorno sobre o investimento, custo padrão e análise de variância; além de medidas não financeiras relacionadas aos clientes. Isso serve para analisar as relações entre as variáveis, estabelecendo como os gestores decidem na prática (Worren, Moore, \& Cardona, 2002) e se estão apoiados em critérios e subcritérios de análise identificados (Li \& Liao, 2007). Tem também como finalidade auxiliar os gestores a tomar decisões por meio de alternativas, visando solucionar o problema avaliado (Hsu, Li, \& Chen, 2010). Alguns modelos resultam também em um conjunto de alternativas reais que podem se alterar durante a construção, bem como gerar novas alternativas (Carl \& Chris, 2003; Hu, Lee, Yen, \& Tsai, 2009; Janssen et al., 2005; Selden \& Sowa, 2004; Wouters \& Wilderom, 2008).

No último grupo estão inseridos os instrumentos de criação de valor, com o uso eficaz dos recursos. Guilding, Cravens e Tayles (2000) classificam como características da análise estratégica a preocupação com os clientes e o ambiente externo, o foco nos concorrentes e a orientação para longo prazo, voltada ao futuro.

As pesquisas realizadas sobre os estágios da CG (Frezatti, 2005; Grande \& Beuren, 2011; Miranda, Carvalho, Libonati, \& Cavalcante, 2013; Pavlatos \& Paggios, 2009; Soutes \& Guerreiro, 2007; Sulaiman, Nazli, \& Alwi, 2004) relatam a predominância do uso das práticas consideradas tradicionais, ou seja, de primeiro e segundo estágio.

\section{Estudos sobre práticas de contabilidade gerencial em hotéis}

No Brasil, em 2016, havia 31,3 mil estabelecimentos de hospedagem, com 1 milhão de unidades habitacionais (suítes, quartos, chalés) e 2,4 milhões de leitos. Entre esses estabelecimentos, 47,9\% eram hotéis, 31,9\% eram pousadas e 14,2\% eram motéis ("Brasil tem 2,4 milhões", 2017). 
A tendência, nos próximos anos, é que haja um aumento substancial da presença das redes hoteleiras em atuação no país. Até meados da década passada, não mais de 30 cidades no Brasil eram atendidas por redes profissionais e, no processo de expansão hoteleira em curso, mais de 200 cidades devem ser contempladas, em todas as regiões brasileiras (Jacob, 2014).

No Brasil, o turismo contribui para 3,5\% do produto interno bruto (PIB), com uma injeção na economia de US\$ 77,6 bilhões (R\$ 166,1 bilhões). 0 turismo gerou 3,1\% dos empregos diretos no país e aproximadamente 8,4 milhões de empregos diretos e indiretos em 2013 (“Economia do turismo", 2017).

Dada a relevância econômica desse ramo para o Brasil, torna-se crucial compreender a dimensão, os conceitos e as especificidades das empresas que estão diretamente relacionadas ao turismo, como as hoteleiras, bem como compreender sua dimensão socioeconômica.

Quanto a seus conceitos e especificidades, no Brasil, os meios de hospedagem são classificados em sete tipos: hotel, resort, hotel fazenda, cama e café, hotel histórico, pousada e flat/apart-hotel ("Economia do turismo", 2017). Essa classificação é regulamentada pela Portaria no 100/2011 do Ministério do Turismo, no seu art. 7ํ ("Portaria no 100", 2011) e pela Portaria Inmetro no 273/2011 do Ministério do Desenvolvimento, Indústria e Comércio Exterior ("Portaria Inmetro/MDIC no 273", 2011).

O Ministério do Turismo ("Economia do turismo", 2017) considera que cada tipo de meio de hospedagem reflete diferentes práticas de mercado e expectativas distintas dos turistas - um hotel cinco estrelas é diferente de uma pousada cinco estrelas, por exemplo. Assim, cada meio de hospedagem, para receber sua classificação, deve atender a um conjunto de requisitos.

A dimensão socioeconômica das empresas hoteleiras responsabiliza a gestão como fator decisivo do sucesso, o que, dada a sofisticação organizacional da sociedade e a constante evolução das tecnologias da informação e comunicação, determina a necessidade de um sistema de informação adequado. Diante desse contexto, as boas práticas de gestão dependem da qualidade do sistema de informação e, no caso das empresas hoteleiras, essa dependência assume especial relevância pelas especificidades desse setor (Lima Santos et al., 2016)

Estudos de práticas de CG aplicados a empresas hoteleiras têm sido realizados tanto na área do turismo como na de contabilidade. Estes estudos abordam aspectos da indústria do turismo e concentram-se principalmente em hotéis (Harris \& Brown, 1998).

Diferentes estudos sobre práticas de orçamento foram realizados entre os anos de 1980 e 2000. Entre eles, podem-se destacar o de Schmidgall e Ninemeier (1987), feito nas 47 maiores cadeias de hotéis dos Estados Unidos; o de Schmidgall, Borchgrevink e Zahl-Begnum (1996), em empresas hoteleiras dos Estados Unidos e da Escandinávia; o de Schmidgall e DeFranco (1998), em hotéis dos Estados Unidos; e o de Jones (1998), em hotéis do Reino Unido.

0 estudo realizado por Atkinson e Brown (2001) mostra que hotéis do Reino Unido utilizam medidas tracionais de desempenho, como as financeiras. A análise dos resultados obtidos sugere que a indústria hoteleira desse país não enfatiza as dimensões de desempenho não financeiras, como a qualidade do serviço e satisfação do cliente, mas sim as dimensões de desempenho financeiro. 
Lima, Egito e Silva (2004) realizaram um estudo envolvendo os hotéis de médio e grande porte do Rio Grande do Norte. Entre os resultados, destaca-se que $62,5 \%$ dos hotéis utilizam o método de custeio por absorção, e $25 \%$ usam o método de custeio variável. Quando questionados acerca da utilização de um sistema de informação no hotel, 65,63\% afirmam não possuir ou desconhecer essa ferramenta. Isso revela que os hotéis da amostra possuem um baixo conhecimento de técnicas gerenciais modernas.

Haktanir e Harris (2005), que exploraram as práticas de medição de desempenho em um hotel no Chipre, verificaram seis temas principais, os quais são agrupados sob a dinâmica empresarial e o desempenho geral, o desempenho dos funcionários, a satisfação dos clientes, o desempenho financeiro e as atividades inovadoras de medida de desempenho.

No estudo feito por Leitão e Silva (2009), foi verificado que o setor hoteleiro no Nordeste do Brasil utiliza sistemas de custos tradicionais. Entre os principais resultados do estudo, pode-se destacar que aproximadamente $72 \%$ dos hotéis analisados utilizam como ferramenta gerencial o custeio por absorção, enquanto $24,5 \%$ utilizam o método de custeio variável, e 3,4\% o custeio por atividades (ABC).

Pavlatos e Paggios (2009) estudaram práticas de CG em hotéis na Grécia. Os resultados mostram que esses estabelecimentos apresentam baixa utilização de sistemas de custos. A maioria desses sistemas não fornecem informações de qualidade, visto que não calculam variações entre orçados e realizados e não fornecem informações detalhadas de custo, concentrando-se no emprego de medidas financeiras tradicionais para a avaliação de desempenho. No entanto, os hotéis estão começando a usar medidas não financeiras em relação aos clientes, à inovação e a funcionários. 0 estudo também mostrou que a utilização do orçamento de base zero era significativo, porém a aplicação do orçamento flexível e por atividades era bastante baixa. 0 alinhamento entre o planejamento estratégico e o orçamento foi considerado baixo.

O estudo de Steed e Gu (2009) foi realizado em hotéis dos Estados Unidos. Os resultados do estudo mostram que o orçamento é amplamente utilizado pelas empresas hoteleiras. Entre as informações utilizadas está o Revenue per Avaliable Room (RevPAR) e o aumento porcentual em relação ao ano anterior. 0 orçamento era elaborado em planilha eletrônica, com disposição mensal dos dados.

Lunkes (2009) pesquisou um universo de 150 hotéis (com 60 respostas) na cidade de Florianópolis e revelou que 24 deles utilizam algum sistema de custos. Os métodos de custeamento mais utilizados foram o por atividades (ABC) (50\%) e o direto ou variável (45\%).

Uyar e Bilgin (2011) realizaram um estudo sobre os hotéis da região de Antália, na Turquia. A pesquisa foi aplicada a 300 hotéis com diferentes características de tamanho e categoria, obtendo 79 respostas. Entre os resultados, pode-se destacar a utilização do orçamento e de informações financeiras para avaliar o desempenho das empresas hoteleiras.

Um estudo realizado por Souza e Lunkes (2015) explora as práticas orçamentárias em hotéis dos principais centros turísticos do Brasil. Os resultados apontam que a maioria dos hotéis utiliza o orçamento empresarial. 0 orçamento é elaborado com frequência pelos hotéis, utilizando-se dos resultados de anos anteriores e de indicadores econômicos. 


\section{METODOLOGIA}

Com relação ao enquadramento metodológico, esta pesquisa caracteriza-se como descritiva quanto a seu objetivo, visto que tem como finalidade identificar as práticas de CG utilizadas pelos hotéis da grande Florianópolis. Quanto à abordagem, é classificada como qualitativa, por se utilizar da análise descritiva dos resultados. Quanto aos procedimentos, classifica-se como um levantamento (survey), realizado por meio da aplicação de um questionário.

A população desse estudo é composta de 55 hotéis filiados à Associação Brasileira da Indústria de Hotéis de Santa Catarina (ABIH), em Florianópolis. 0 contato com os estabelecimentos foi feito via e-mail, ocasião em que foram expostos a natureza e o objetivo da pesquisa, e os pesquisados foram convidados a responder ao questionário encaminhado por e-mail, disponibilizado na plataforma Google Docs. Da população inicial de hotéis, foram obtidas 15 devolutivas, sendo os questionários respondidos pelo controller ou gerente do hotel, entre o período de 26 de abril de 2015 e 4 de maio de 2016. Portanto o estudo limita-se a 15 hotéis situados na grande Florianópolis e, desta forma, os resultados refletem este escopo e não podem ser generalizados.

0 instrumento da pesquisa constitui-se de 64 questões assertivas, nas quais foi solicitado que os entrevistados indicassem se o hotel adotava práticas de CG e, em caso positivo, quais; em seguida, caso essas práticas fossem utilizadas, pedia-se que listassem os benefícios obtidos ao longo dos últimos três anos. Os entrevistados também foram convidados a classificar o grau de ênfase que o hotel daria para cada prática ao longo dos próximos três anos. A escala utilizada foi de 1 a 5 pontos, sendo o grau 1 correspondente a nenhum benefício, e o grau 5 a um alto benefício em relação aos últimos anos e aos próximos três. 0 questionário foi elaborado com base na literatura levantada, conforme o Quadro 2.

Quadro 2 - Conteúdo do instrumento de pesquisa

\begin{tabular}{|c|c|}
\hline Assunto & Referências \\
\hline Contabilidade de custos & $\begin{array}{l}\text { Bjornenak (1997); Bjornenak e Mitchell (1999); Drury et al. (1993); } \\
\text { Garrison e Noreen (2003); Lucas (1997); Lunkes (2009); } \\
\text { e Zimmerman (2000). }\end{array}$ \\
\hline Orçamento & $\begin{array}{l}\text { Atkinson e Brown (2001); Castro et al. (2012); Drury (2000); } \\
\text { Hansen e Mowen (2002); Horngren, Bhimani, Datar, \& Foster } \\
\text { (2002); Hilton (2002); Souza e Lunkes (2015); Steed e Gu (2009); } \\
\text { e Uyar e Bilgin (2011). }\end{array}$ \\
\hline $\begin{array}{l}\text { Avaliação de } \\
\text { desempenho }\end{array}$ & $\begin{array}{l}\text { Elnathan et al. (1996); Ittner, Larcker, \& Rajan (1997); Kaplan e } \\
\text { Norton (1997); McNair e Leibfried (1992); e Shields (1997). }\end{array}$ \\
\hline $\begin{array}{l}\text { Análise utilizada para } \\
\text { tomada de decisão }\end{array}$ & $\begin{array}{l}\text { Abdel-Kader e Luther (2006); Abernethy et al. (2005); Aliguliyev } \\
\text { (2009); Balasubramanian e Gupta (2005); Carl e Chris (2003); } \\
\text { Chin et al. (2010); Drury (2000); Hansen e Mowen (2002); Hilton } \\
\text { (2002); Hsu et al. (2010); Hu et al. (2009); Ittner \& Larcker (1998); } \\
\text { Janssen et al. (2005); Li e Liao (2007); Needles e Crosson (2002); } \\
\text { Nudurupati et al. (2011); Selden e Sowa (2004); Worren, Moore e } \\
\text { Cardona (2002); e Wouters e Wilderom (2008). }\end{array}$ \\
\hline Análise estratégica & Abdel-Kader e Luther (2006); e Guilding et al. (2000). \\
\hline
\end{tabular}

Fonte - Atualizado a partir de Pavlatos e Paggios (2009) 
Os dados foram analisados a partir de uma análise descritiva das informações obtidas pelo questionário, com auxílio do software Excel.

\section{DESCRIÇÃo E ANÁLISE DOS DADOS}

A seguir, apresenta-se a descrição do perfil das empresas respondentes, a análise descritiva dos dados das práticas adotadas por hotéis da grande Florianópolis, assim como os graus de benefícios passados e futuros das práticas de CG, nos últimos e nos próximos três anos.

\section{O perfil das empresas hoteleiras}

Na Tabela 1, evidencia-se o perfil dos 15 hotéis respondentes da pesquisa, com destaque para a categoria, o número de leitos, a gestão da empresa e o tipo do hotel.

Tabela 1 - Dados demográficos dos hotéis participantes da pesquisa

\begin{tabular}{|l|c|}
\hline Categorias & Quantidade \\
\hline 1 estrela & 3 \\
\hline 3 estrelas & 4 \\
\hline 5 estrelas & 1 \\
\hline Sem classificação & 5 \\
\hline Outro & 2 \\
\hline Tipo do hotel & \\
\hline Hotel urbano & 10 \\
\hline Flat/Apart-Hotel & 2 \\
\hline Pousada & 3 \\
\hline Gestão da empresa & \\
\hline Empresa privada & 10 \\
\hline Membro de rede nacional & 2 \\
\hline Membro de rede multinacional & 2 \\
\hline Outra & 1 \\
\hline Número de camas & 1 \\
\hline Até 100 & \\
\hline De 101 a 200 & 9 \\
\hline De 301 a 400 & 3 \\
\hline Mais de 400 & \\
\hline
\end{tabular}

Na Tabela 1, destaca-se que a maioria dos hotéis não possui classificação em alguma categoria (sem classificação), o que se deve, em parte, ao fato de serem empresas de pequeno porte, tendo em vista que possuem até 100 unidades habitacionais. Em relação à gestão da empresa e ao tipo, aproximadamente $67 \%$ são privadas e hotéis urbanos, ou seja, não são membros de grandes cadeias de hotéis e estão localizadas no centro urbano. 


\section{Resultado da adoção de práticas de contabilidade gerencial}

Nesta seção observam-se as práticas de CG adotadas pelas empresas hoteleiras. Os resultados obtidos estão evidenciados na Tabela 2.

Tabela 2 - Práticas de contabilidade gerencial adotadas

\begin{tabular}{|c|c|}
\hline & Quantidade \\
\hline \multicolumn{2}{|l|}{ Método de custeio } \\
\hline Custeio por absorção & 7 \\
\hline Custeio variável & 1 \\
\hline Custeio baseado em atividades (ABC) & 0 \\
\hline Custeio padrão & 7 \\
\hline \multicolumn{2}{|l|}{ Práticas de orçamento } \\
\hline Planejamento anual & 12 \\
\hline Para controle de custos & 6 \\
\hline Para coordenar atividades das diversas áreas da organização & 1 \\
\hline Para avaliar o desempenho dos gestores & 0 \\
\hline Orçamento base zero & 1 \\
\hline Orçamento em longo prazo & 3 \\
\hline Orçamento flexível & 1 \\
\hline Orçamento baseado em atividades & 3 \\
\hline \multicolumn{2}{|l|}{ Tipo de análise utilizada para tomada de decisão } \\
\hline Análise de rentabilidade do produto & 10 \\
\hline Análise de rentabilidade do cliente & 11 \\
\hline Análise do ciclo de vida do produto (CVP) & 3 \\
\hline \multicolumn{2}{|l|}{ Método de avaliação de desempenho } \\
\hline $\begin{array}{l}\text { Medidas de rentabilidade (lucro operacional e aumento de } \\
\text { receitas) }\end{array}$ & 11 \\
\hline Medidas não financeiras relacionadas aos clientes & 4 \\
\hline Medidas não financeiras relacionadas a inovações & 0 \\
\hline Medidas não financeiras relacionadas a empregados & 0 \\
\hline Retorno sobre investimento (ROI) & 2 \\
\hline Receita residual & 0 \\
\hline Valor econômico agregado (EVA) & 1 \\
\hline Retorno sobre vendas & 2 \\
\hline Balanced scorecard & 0 \\
\hline Benchmarking & 1 \\
\hline \multicolumn{2}{|l|}{ Tipo de análise estratégica utilizada } \\
\hline Análise de indústria & 6 \\
\hline Análise dos pontos fortes e fracos dos concorrentes & 11 \\
\hline Análise da posição dos concorrentes & 9 \\
\hline Previsão de longo alcance & 1 \\
\hline Gestão baseada em atividades & 3 \\
\hline
\end{tabular}

Fonte - elaboração dos autores 
Pode-se observar, na Tabela 2, que as empresas hoteleiras utilizam como sistema de custos o custeio por absorção e o custeio padrão. Esses dados são congruentes com os resultados do estudo de Pavlatos e Paggios (2009), no qual a maioria dos hotéis gregos utilizam esses métodos de custeio. Esses resultados também são coerentes com estudos nacionais, como o de Lima, Egito e Silva (2004) no Rio Grande do Norte, que apontam 62,5\% de utilização do custeio por absorção, e o de Leitão e Silva (2009) no Nordeste do Brasil, com uso desse método por $72 \%$ dos hotéis.

Com relação à prática de orçamento, verifica-se que a maioria dos hotéis utiliza técnicas de orçamento tradicionais, sendo que 12 utilizam o orçamento com o objetivo de realizar o planejamento anual, e 6 para controle de custos. Estudos internacionais e nacionais também relatam a utilização do orçamento (Pavlatos \& Paggios, 2009; Souza \& Lunkes, 2015; Steed \& Gu, 2009; Uyar \& Bilgin; 2011). A pesquisa de Pavlatos e Paggios (2009) mostra ainda que o orçamento flexível e por atividades é pouco utilizado nos hotéis gregos, com exceção do orçamento de base zero, que tem uso significativo. Os orçamentos tradicionais atuam de forma satisfatória em ambientes estáveis; no entanto, em ambientes altamente dinâmicos, perdem sua eficácia (Vanzella \& Lunkes, 2009).

As medidas de avaliação de desempenho mais adotadas pelas empresas hoteleiras são as de rentabilidade financeira, sendo que quatro hotéis utilizam medidas não financeiras relacionadas com clientes. A utilização de medidas financeiras na avaliação de desempenho parece ser uma prática comum nas empresas hoteleiras, conforme apontado nos estudos de Atkinson e Brown (2001), Haktanir e Harris (2005), Pavlatos e Paggios (2009), Steed e Gu (2009), Uyar e Bilgin (2011) e Souza e Lunkes (2015), embora a tomada de decisão com base em informações financeiras seja criticada (Ittner et al., 1997; Kaplan \& Norton, 1997) por promover uma visão em curto prazo (Abdel-Kader \& Luther, 2006). Os resultados desta pesquisa mostram que os hotéis ainda não incorporaram o uso de informações não financeiras.

As evidências do estudo revelam que os tipos de análise utilizados para a tomada de decisão são a análise de rentabilidade do cliente, feita por 11 hotéis, seguida da análise de rentabilidade do produto, feita por 10 empresas hoteleiras. Isso mostra que os gestores têm grande preocupação com a lucratividade e a ocupação das unidades habitacionais, o que se justifica, pois a receita de um quarto não ocupado está perdida e não pode ser recuperada no dia seguinte.

Os tipos de análise estratégica adotados são a análise dos pontos fortes e fracos dos concorrentes (11), da posição dos concorrentes (9) e a análise de indústria (6). Dessa forma, pode-se concluir que os hotéis da amostra têm preocupação com possíveis vantagens competitivas e com sua posição no mercado em relação aos concorrentes. A Tabela 3 apresenta os graus de benefícios passados e futuros das práticas de CG, considerando os últimos e os próximos três anos.

Verifica-se, na Tabela 3, que os respondentes avaliam que há alto grau de benefício, tanto nos últimos três anos quanto nos próximos, nas técnicas tradicionais de método de custeio, como o custeio por absorção e o custeio padrão. A Tabela 3 mostra também que os respondentes consideram que práticas como o custeio $\mathrm{ABC}$ e o custeio variável têm um baixo grau de benefício. Pelas características das empresas hoteleiras, com altos custos indiretos e fixos (Lima Santos et al., 2016), é relativamente baixa a utilização do custeio variável, considerando a 
participação deste na composição dos custos totais - o que torna a informação de custos variáveis irrelevante no processo de tomada de decisão para os gestores hoteleiros, contribuindo também para sua baixa expectativa de utilização futura.

Tabela 3 - Grau de benefícios passados e futuros das práticas de contabilidade gerencial

\begin{tabular}{|c|c|c|c|c|c|c|}
\hline \multirow{2}{*}{ Grau de benefício } & \multicolumn{3}{|c|}{ Últimos três anos } & \multicolumn{3}{|c|}{ Próximos três anos } \\
\hline & Alto & Médio & Baixo & Alto & Médio & Baixo \\
\hline \multicolumn{7}{|l|}{ Método de custeio } \\
\hline Custeio por absorção & 7 & 3 & 5 & 8 & 2 & 5 \\
\hline Custeio variável & 1 & 3 & 8 & 1 & 3 & 9 \\
\hline Custeio baseado em atividades (ABC) & 0 & 2 & 9 & 0 & 2 & 11 \\
\hline Custeio padrão & 7 & 1 & 2 & 7 & 1 & 5 \\
\hline \multicolumn{7}{|l|}{ Práticas de orçamento } \\
\hline Planejamento anual & 12 & 1 & 1 & 12 & 1 & 1 \\
\hline Para controle de custos & 6 & 4 & 3 & 6 & 4 & 4 \\
\hline $\begin{array}{l}\text { Para coordenar atividades das } \\
\text { diversas áreas da organização }\end{array}$ & 1 & 4 & 6 & 1 & 4 & 6 \\
\hline $\begin{array}{l}\text { Para avaliar o desempenho dos } \\
\text { gestores }\end{array}$ & 0 & 2 & 9 & 0 & 4 & 7 \\
\hline Orçamento base zero & 1 & 1 & 9 & 1 & 3 & 7 \\
\hline Orçamento em longo prazo & 3 & 1 & 8 & 3 & 3 & 5 \\
\hline Orçamento flexível & 1 & 8 & 5 & 1 & 8 & 5 \\
\hline Orçamento baseado em atividades & 3 & 1 & 8 & 3 & 1 & 8 \\
\hline \multicolumn{7}{|l|}{ Método de avaliação de desempenho } \\
\hline $\begin{array}{l}\text { Medidas de rentabilidade (lucro } \\
\text { operacional e aumento de receitas) }\end{array}$ & 11 & 5 & 3 & 12 & 0 & 3 \\
\hline $\begin{array}{l}\text { Medidas não financeiras relacionadas } \\
\text { aos clientes }\end{array}$ & 4 & 7 & 4 & 3 & 7 & 2 \\
\hline $\begin{array}{l}\text { Medidas não financeiras relacionadas } \\
\text { a inovações }\end{array}$ & 0 & 3 & 9 & 6 & 4 & 6 \\
\hline $\begin{array}{l}\text { Medidas não financeiras relacionadas } \\
\text { a empregados }\end{array}$ & 0 & 0 & 10 & 1 & 3 & 9 \\
\hline Retorno sobre investimento (ROI) & 2 & 0 & 11 & 2 & 0 & 10 \\
\hline Receita residual & 0 & 2 & 11 & 0 & 3 & 10 \\
\hline Valor econômico agregado (EVA) & 1 & 0 & 9 & 1 & 0 & 11 \\
\hline Retorno sobre vendas & 2 & 4 & 7 & 3 & 3 & 6 \\
\hline Balanced scorecard & 0 & 1 & 11 & 0 & 1 & 11 \\
\hline Benchmarking & 1 & 0 & 11 & 1 & 0 & 11 \\
\hline \multicolumn{7}{|c|}{ Tipo de análise utilizada para tomada de decisão } \\
\hline Análise de rentabilidade do produto & 10 & 1 & 4 & 10 & 1 & 4 \\
\hline Análise de rentabilidade do cliente & 11 & 3 & 0 & 11 & 3 & 0 \\
\hline $\begin{array}{l}\text { Análise do ciclo de vida do produto } \\
\text { (CVP) }\end{array}$ & 3 & 4 & 6 & 3 & 3 & 7 \\
\hline
\end{tabular}


Tabela 3 - Continuação

\begin{tabular}{|l|c|c|c|c|c|c|}
\hline \multicolumn{1}{|c|}{ Grau de benefício } & \multicolumn{3}{|c|}{ Últimos três anos } & \multicolumn{3}{c|}{ Próximos três anos } \\
\hline & Alto & Médio & Baixo & Alto & Médio & Baixo \\
\hline Tipo de análise estratégica utilizada & & & & & \\
\hline \\
\hline Análise de indústria & 6 & 6 & 1 & 5 & 7 & 1 \\
\hline $\begin{array}{l}\text { Análise dos pontos fortes e fracos dos } \\
\text { concorrentes }\end{array}$ & 11 & 2 & 1 & 7 & 5 & 2 \\
\hline Análise da posição dos concorrentes & 9 & 2 & 0 & 5 & 4 & 2 \\
\hline \begin{tabular}{l} 
Previsão de longo alcance \\
\hline Gestão baseada em atividades (ABM)
\end{tabular} & 1 & 7 & 6 & 0 & 4 & 6 \\
\hline
\end{tabular}

Fonte - Elaboração dos autores

0 resultado que surpreende é o da baixa utilização atual e futura do custeio $\mathrm{ABC}$, pois este é considerado um método apropriado para as características das empresas de serviços, como é o caso dos hotéis. A utilização de métodos de custos mais tradicionais, como o custeio por absorção e o custeio padrão, adaptados das indústrias, pode apresentar restrições quanto à qualidade da informação. Isso também é relatado no estudo de Pavlatos e Paggios (2009) em hotéis na Grécia, em que a maioria desses sistemas não fornecia informações de qualidade, como variações entre orçado e realizado e o detalhamento do custo. Isso pode ocorrer porque, em geral, esses métodos utilizam muitas bases arbitrárias de rateio para o cálculo do custo, o que pode comprometer o resultado final da informação.

Com relação à prática de orçamento, a maioria dos respondentes atribuem alto grau de benefício às técnicas de orçamento tradicionais, ou seja, o planejamento anual e o controle de custos. 0 orçamento parece ser uma prática de CG muito disseminada e utilizada em empresas hoteleiras (Pavlatos \& Paggios, 2009; Uyar \& Bilgin, 2011), dada a necessidade que estas têm de planejar sua demanda e os reflexos que essa projeção tem sobre os resultados. A possibilidade de utilização de técnicas mais sofisticadas, como o orçamento de base zero e por atividades, parece ser pequena no futuro. Especificamente em relação ao orçamento de base zero, não há perspectivas de grandes avanços. A utilização desse processo orçamentário, em particular, poderia contribuir para a gestão hoteleira, considerando sua necessidade de justificar novos gastos, o que cria uma atitude interrogativa em vez de assumir práticas decorrentes do passado (Lunkes, 2007; Pyhrr, 1981).

Percebe-se ainda que as medidas financeiras continuarão sendo importantes ao longo dos próximos três anos, visto que 12 dos respondentes consideram altos os benefícios da utilização de medidas de rentabilidade (lucro operacional e aumento de receitas). Destaca-se que seis dos respondentes avaliam como altos os benefícios das medidas não financeiras relacionadas a inovações, e quatro como médios. Isso revela que os respondentes estão começando a considerar as medidas não financeiras relevantes para a avaliação de desempenho, conforme os achados de Pavlatos e Paggios (2009).

O que surpreende nestes resultados é a baixa utilização de informações sobre os clientes, pois com o avanço da internet e a utilização de sites de reservas (por exemplo, Trivago, Booking, TripAdvisor etc.) os clientes têm acesso às avaliações de outros hóspedes, informações que podem influenciar as decisões de compra 
(Gretzel \& Yoo, 2008). Assim, ao utilizar essas ferramentas, o gestor tende a identificar oportunidades de melhoria no serviço e a aumentar a qualidade da experiência dos clientes (Kim, Kim, \& Heo, 2016), o que passa a ser uma fonte de informação importante para os gestores hoteleiros e, de certa forma, uma preocupação. Esses resultados estão refletidos na baixa utilização de ferramentas como o balanced scorecard e o benchmarking, que poderiam auxiliar os hotéis na gestão dessas informações não financeiras.

Com relação ao tipo de análise estratégica, a análise de rentabilidade do produto (10) e a análise de rentabilidade do cliente (11) se mantêm como as mais benéficas nos últimos e nos próximos três anos, de acordo com os entrevistados.

Por fim, o tipo de análise estratégica considerado mais benéfico para os próximos três anos continua a ser a análise dos pontos fortes e fracos dos concorrentes (7). Destaca-se também o aumento do grau de benefícios futuros da gestão baseada em atividades (ABM).

\section{CONSIDERAÇÕES FINAIS}

Esse estudo teve como objetivo analisar as práticas de CG em empresas hoteleiras da grande Florianópolis. Para tanto, foram coletados dados de 15 hotéis. Os resultados mostram a utilização de práticas de custos tradicionais, como o custeio por absorção e o custeio padrão, ambos apropriados das empresas industriais. Parecem ser práticas consolidadas nas empresas hoteleiras, já que estudos anteriores também destacaram esses métodos de custeio, o que mostra pouca evolução nas últimas décadas. Ou seja, as práticas são as mesmas, assim como não parece haver interesse em modificá-las nos próximos três anos.

Da mesma forma que os métodos de custeio, as práticas de orçamento também são relativamente tradicionais, com a utilização do orçamento anual para controle de custos, embora estudos internacionais mostrem que hotéis em diferentes partes do mundo têm experimentado outras técnicas, como o orçamento de base zero (Jones, 1998; Pavlatos \& Paggios, 2009). Os gestores não têm intenção de modificar essa realidade nos próximos três anos.

A utilização de medidas financeiras é uma prática que se destaca nos resultados desta pesquisa e nos estudos anteriores. Embora estudos mostrem que as medidas não financeiras também são importantes, os gestores se sentem mais confortáveis ao tomar decisões munidos com informações sobre o lucro, o aumento de receita, indicadores como o RevPAR etc. Nessas práticas há um ponto de flexão: a utilização de medidas não financeiras relacionadas à inovação nos próximos três anos. Isso mostra a preocupação dos gestores com a manutenção e a atualização do hotel, visando manter sua capacidade produtiva e seus níveis de ocupação.

$\mathrm{Na}$ análise para tomada de decisão e na análise estratégica, três pontos foram destacados: a análise de rentabilidade do produto, do cliente e dos concorrentes. Essas informações são centrais na gestão hoteleira, assim como conhecer a rentabilidade por tipo produto (habitação, bar e alimentação, garagem, salão de eventos etc.), habitação (standard, duplo, triplo, suíte presidencial etc.) e cliente. Aqui também os gestores apontaram uma mudança significativa para os próximos três anos, que é a adoção da gestão baseada em atividades. 
Esses resultados sugerem uma preferência por práticas de gestão tradicionais, incluídas nos estágios 1 e 2, com ênfase na determinação dos custos, no controle financeiro e na provisão de informação para planejamento e controle gerencial. Não parece haver um clima de mudança em vista, já que os gestores hoteleiros não demostraram grandes intenções de inovar nas práticas de gestão nos próximos três anos.

Em relação a estudos futuros, pode-se entender melhor as possíveis razões da utilização de práticas tradicionais, ou seja, os fatores que podem explicar os resultados encontrados. Além disso, ainda é importante compreender melhor como as práticas são adotadas, seus níveis de implementação e utilização, entre outras possibilidades de pesquisa neste tema.

\section{REFERÊNCIAS}

Abdel-Kader, M. G., \& Luther, R. G. (2006). IFAC's conception of the evolution of management accounting. Advances in management accounting, 15, 229-247. doi:10.1016/S14747871(06)15010-8

Abernethy, M. A., Horne, M., Lillis, A. M., Malina, M. A., \& Selto, F. J. (2005). A multi-method approach to building causal performance maps from expert knowledge. Management Accounting Research, 16(2), 135-155. doi:10.1016/j.mar.2005.03.003

Aliguliyev, R. M. (2009). Performance evaluation of density-based clustering methods. Information Sciences, 179(20), 3583-3602. doi:10.1016/j.ins.2009.06.012

Atkinson, H., \& Brown, J. B. (2001). Rethinking performance measures: Assessing progress in UK hotels. International Journal of Contemporary Hospitality Management, 13(3), 128-136. doi:10.1108/09596110110388918

Bain \& Company. (2013). Management tools \& trends. Recuperado de https://bit.ly/OBO3i6

Balasubramanian, S., \& Gupta, M. (2005). Structural metrics for goal based business process design and evaluation. Business Process Management Journal, 11(6), 680-694. doi:10.1108/14637150510630855

Bjornenak, T. (1997). Diffusion and accounting: The case of ABC in Norway. Management Accounting Research, 8(3), 3-14. doi:10.1006/mare.1996.0031

Bjornenak, T., \& Mitchell, F. (1999). A study of the development of the activity-based costing journal literature 1987-1998. Working paper, University of Edinburgh, Edinburgh.

Brasil tem 2,4 milhões de leitos em sua rede de hospedagem. (2017). Agência IBGE Notícias. Recuperado de https://bit.ly/2Gc9ULe

Carl, O., \& Chris, D. (2003). Packaging scorecard: A packaging performance evaluation method. Packaging Technology and Science, 16(1), 9-14. doi:10.1002/pts.604

Castro, J. K., De Souza, P., Rockenbach, G. S., \& Lunkes, R. J. (2012). Prácticas presupuestarias aplicadas a la empresa hotelera de Brasil: Un estudio de la ciudad de Florianópolis. Estudios y Perspectivas en Turismo, 21(4), 904-924.

Chin, K. S., Lo, K. C., \& Leung, J. P. F. (2010). Development of user-satisfaction-based knowledge management performance measurement system with evidential reasoning approach. Expert Systems with Applications, 37(1), 366-382. doi:10.1016/j.eswa.2009.05.051

Codesso, M. M., Lunkes, R. J., \& Suave, R. (2013). Práticas orçamentárias aplicadas em empresas hoteleiras no Brasil: Um estudo na cidade de Balneário Camboriú - SC. Revista Turismo Visão e Ação, 15(2), 279-294. doi:10.14210/rtva.v15n2.p279-294 
Deboleto, G. A. G., Hall, R. J., Dutra, F. M., \& Silva, R. J. (2013). Análise do uso do orçamento empresarial em uma empresa de pequeno porte: Um estudo de caso um comércio de iluminação. Comunicação \& Mercado/Unigran, 1(4), 98-114. Recuperado de https://bit. ly/2uVu0mv

Drury, C. (2000). Management and cost accounting. London, UK: Thomson Learning Europe.

Drury, J. C., Braund, S., Osborne, P., \& Tayles, M. (1993). A survey of management accounting practices in UK manufacturing companies. London, UK: Certified Accountants Educational Trust.

Economia do turismo cresce no Brasil. (2017). Ministério do Turismo. Recuperado de https://bit.ly/2UHc0em

Frezatti, F. (2005). Management accounting profile of firms located in Brazil: A field study. Brazilian Administration Review, 2(1), 73-87. doi:10.1590/S1807-76922005000100006

Garrison, R. H., \& Noreen, E. W. (2003). Managerial accounting. New York, NY: McGraw-Hill.

Grande, J. F., \& Beuren, I. M. (2011). Mudanças de práticas de contabilidade gerencial: Aplicação da análise de discurso crítica no relatório da administração de empresa familiar. BASE - Revista de Administração e Contabilidade da Unisinos, 8(2), 133-145. doi:10.4013/ base.2011.82.03

Gretzel, U, \& Yoo, K. H. (2008). Use and impact of online travel reviews. In P. O'Connor, W. Höpken, U. Gretzel (Eds.), Information and communication technologies in tourism (pp. 3546). Vienna: Springer.

Guilding, C., Cravens, K. S., \& Tayles, M. (2000). An international comparison of strategic management accounting practices. Management Accounting Research, 11(1), 113-135. doi:10.1006/mare.1999.0120

Gurgel, G. (2016). Sol e praia são a principal atração de estrangeiros para o Brasil. Ministério do Turismo. Recuperado de https://bit.ly/2uXmlUJ

Haktanir, M., \& Harris, P. (2005). Performance measurement practice in an independent hotel context: A case study approach. International Journal of Contemporary Hospitality Management, 17(1), 39-50. doi:10.1108/09596110510577662

Hansen, D. R., \& Mowen, M. M. (2002). Management accounting. Cincinnati, OH: SouthWestern College Publishing.

Harris, P. J., \& Brown, J. B. (1998). Research and development in hospitality accounting and financial management. International Journal of Hospitality Management, 17(2), 161-182. doi:10.1016/S0278-4319(98)00013-9

Hilton, R. W. (2002). Managerial accounting: Creating value in a dynamic business environment. New York, NY: McGraw-Hill Irwin.

Horngren, C. T., Sundem, G. L., \& Stratton, W. O. (2004). Contabilidade gerencial. São Paulo, SP: Prentice Hall.

Horngren, C. T., Bhimani, A., Datar, S. M., \& Foster, G. (2002). Management and cost accounting. London, UK: Financial Times Prentice Hall.

Hsu, Y. L., Li, W. C., \& Chen, K. W. (2010). Structuring critical success factors of airline safety management system using a hybrid model. Transportation Research Part E: Logistics and Transportation Review, 46(2), 222-235. doi:10.1016/j.tre.2009.08.005

Hu, H.-Y., Lee, Y.-C., Yen, T-M., \& Tsai, C-H. (2009). Using BPNN and DEMATEL to modify importance-performance analysis model: A study of the computer industry. Expert Systems with Applications, 36(6), 9969-9979. doi:10.1016/j.eswa.2009.01.062

Institute of Management Accountants. (2008). Definition of management accounting. Practice of Management Accounting, pp. 1-4. Recuperado de https://bit.ly/2I6s0AJ 
International Federation of Accountants [Website]. (1998). Recuperado de www.ifac.org Ittner, C. D., \& Larcker, D. F. (1998). Are nonfinancial measures leading indicators of financial performance? An analysis of customer satisfaction. Journal of Accounting Research, 36, 1-35. doi:10.2307/2491304

Ittner, C., Larcker, D., \& Rajan, M. (1997). The choice of performance measures in annual bonus contracts. The Accounting Review, 72(2), 231-55.

Jacob, C. S. C. (2014). Superoferta de hotéis: Panorama do setor hoteleiro brasileiro após a Copa. Construção Mercado: Negócios de Incorporação e Construção, (156). Recuperado de https://bit.ly/2WWWs3u

Janssen, R., Goosen, H., Verhoeven, M. L., Verhoeven, J. T. A., Omtzigt, A. Q. A., \& Maltby, E. (2005). Decision support for integrated wetland management. Environmental Modelling \& Software, 20(2), 215-229. doi:10.1016/j.envsoft.2003.12.020

Jiambalvo, J. (2000). Contabilidade gerencial. Rio de Janeiro, RJ: LTC.

Jones, T. A. (1998). UK hotel operators use of budgetary procedures. International Journal of Contemporary Hospitality Management, 10(3), 96-100. doi:10.1108/09596119810210279

Kaplan, R. S., \& Norton, D. P. (1997). A estratégia em ação: Balanced scorecard. Rio de Janeiro, RJ: Campus.

Kim, B., Kim, S., \& Heo, C. Y. (2016). Analysis of satisfiers and dissatisfiers in online hotel reviews on social media. International journal of contemporary hospitality management, 28(9), 1915-1936. doi:10.1108/IJCHM-04-2015-0177

Leitão, C. R. S., \& Silva, J. D. G. (2009). Utilização do custeio variável no gerenciamento de hotéis: Uma pesquisa no setor hoteleiro do nordeste brasileiro. Contabilidade Vista \& Revista, 17(3), 25-43. Recuperado de https://bit.ly/2KkmgVK

Li, Y., \& Liao, X. (2007). Decision support for risk analysis on dynamic alliance. Decision Support Systems, 42(4), 2043-2059. doi:10.1016/j.dss.2004.11.008

Lima, G. A. S., Egito, M. O. T., \& Silva, J. D. G. (2004). Utilização de informações de custos no processo gerencial: Estudo comparativo entre a hotelaria do estado do Rio Grande do Norte e a região nordeste, sob a ótica da gestão econômico-financeira. Revista Contabilidade \& Finanças, 15, 106-116. doi:10.1590/S1519-70772004000400008

Lima Santos, L., Gomes, C., Faria, A. R., Lunkes, R. J., Malheiros, C., Rosa, F.S., \& Nunes, C. (2016). Contabilidade de gestão hoteleira. Cacém, Portugal: ATF.

Lucas, M. (1997). Standard costing and its role in today's manufacturing environment. Management Accounting, 75(4), 32-4.

Lunkes, R. J. (2007). Manual de orçamento (2 ${ }^{\mathrm{a}}$ ed.). São Paulo, SP: Atlas.

Lunkes, R. J. (2009). Informações de custos: Um estudo em empresas hoteleiras na cidade de Florianópolis - SC. Turismo em Análise, 20(2), 345-368. doi:10.11606/issn.1984-4867. v20i2p345-368

Martins, E. (2003). Contabilidade de custos (9a ed.). São Paulo, SP: Atlas.

Maximiano, A. (2000). Teoria geral da administração: Da escola cientifica à competitividade na economia globalizada (2 ${ }^{\underline{a}}$ ed.). São Paulo, SP: Atlas.

Miranda, L. C., Carvalho, I. R., Libonati, J. J., \& Cavalcante, P. R. N. (2013). Práticas de contabilidade gerencial: Evidências em consultorias oferecidas por revistas especializadas aos empresários das micro, pequenas e médias empresas. Revista Contabilidade e Controladoria, 5(1), 139-157. doi:10.5380/rcc.v5i1.28237

Needles, B. E., \& Crosson, S. V. (2002). Managerial accounting. Boston: Houghton Mifflin Company. 
Nudurupati, S. S., Bititci, U. S., Kumar, V., \& Chan, F. T. (2011). State of the art literature review on performance measurement. Computers \& Industrial Engineering, 60(2), 279290. doi:10.1016/j.cie.2010.11.010

Pavlatos, O., \& Paggios, I. (2009). Management accounting practices in the Greek hospitality industry. Managerial Auditing Journal, 24(1), 81-98. doi:10.1108/02686900910919910

Pellinen, J. (2003). Making price decisions in tourism enterprises. International Journal of Hospitality Management, 22(2), 217-235. doi:10.1016/S0278-4319(03)00019-7

Portaria no 100, de 16 de junho de 2011 (2011, 16 de junho). Ministério do Turismo. Institui o Sistema Brasileiro de Classificação de Meios de Hospedagem (SBClass), cria o Conselho Técnico Nacional de Classificação de Meios de Hospedagem (CTClass) e dá outras providências. Diário Oficial da União, seção 1.

Portaria Inmetro/MDIC no 273, de 21 de junho de 2011 (2011, 21 de junho). Ministério do Desenvolvimento, Indústria e Comércio Exterior. Determinar que a etapa de verificação de acompanhamento do Meio de Hospedagem - MH será iniciada pelo representante técnico do Inmetro, prevista no RAC aprovado pela Portaria Inmetro no 485/2010. Diário Oficial da União, seção 1.

Pyhrr, P. A. (1981). Orçamento base zero. São Paulo, SP: Interciência.

Schmidgall, R. S., Borchgrevink, C. P., \& Zahl-Begnum, O. H. (1996). Operations budgeting practices of lodging firms in the United States and Scandinavia. International Journal of Hospitality Management, 15(2), 189-203. doi:10.1016/0278-4319(96)00008-4

Schmidgall, R. S., \& DeFranco, A. L. (1998). Budgeting and forecasting. The Cornell Hotel and Restaurant Administration Quarterly, 39(6), 45-51. doi:10.1016/S0010-8804(99)80005-6

Schmidgall, R. S., \& Ninemeier, J. D. (1987). Budgeting in hotel chains: Coordination and control. Cornell Hotel and Restaurant Administration Quarterly, 28(1), 78-84. doi:10.1177/001088048702800118

Selden, S. C., \& Sowa, J. E. (2004). Testing a multi-dimensional model of organizational performance: Prospects and problems. Journal of Public Administration Research and Theory, 14(3), 395-416. doi:10.1093/jopart/muh025

Soutes, D. O., \& Guerreiro, R. (2007, setembro). Uma investigação do uso de artefatos da contabilidade gerencial por empresas brasileiras. Trabalho apresentado no XXXI Encontro da Associação Nacional de Pós-Graduação e Pesquisa em Administração, Rio de Janeiro, RJ.

Souza, P., \& Lunkes, R. J. (2015). Práticas de orçamento: Um estudo em empresas hoteleiras do Brasil. Revista Brasileira de Pesquisa em Turismo, 9(3), 380-399. doi:10.7784/rbtur. v9i3.851

Souza, P., Souza, R. M., Petri, S. M., \& Lunkes, R. J. (2015). Development of balanced scorecard as a strategic management for a graduate program. Revista IEEE Latin America, 13(1), 277-283. doi:10.1109/TLA.2015.7040659

Steed, E., \& Gu, Z. (2009). Hotel management company forecasting and budgeting practices: A survey-based analysis. International Journal of Contemporary Hospitality Management, 21(6), 676-697. doi:10.1108/09596110910975954

Sulaiman, M. B., Nazli, N. A., \& Alwi, N. (2004). Management accounting practices in selected Asian countries: A rewiew of the literature. Managerial Auditing Journal, 4(19), 493-508. doi:10.1108/02686900410530501

Uyar, A., \& Bilgin, N. (2011). Budgeting practices in the Turkish hospitality industry: An exploratory survey in the Antalya region. International Journal of Hospitality Management, 30,(2), 398-408. doi:10.1016/j.ijhm.2010.07.011 
Vanzella, C., \& Lunkes, R. J. (2009). Orçamento baseado em atividades: Um estudo de caso em empresa distribuidora de energia elétrica. Contabilidade Vista \& Revista, 17(1), 113132. Recuperado de https://bit.ly/2G1g7sb

Worren, N., Moore, K. E., \& Cardona, P. (2002). Modularity, strategic flexibility, and firm performance: A study of the home appliance industry. Strategic Management Journal, 23(12), 1123-1140. Recuperado de https://bit.ly/2Z0PEUo

Wouters, M., \& Wilderom, C. (2008). Developing performance-measurement systems as enabling formalization: A longitudinal field study of a logistics department. Accounting, Organizations and Society, 33(4-5), 488-516. doi:10.1016/j.aos.2007.05.002

Zimmerman, J. (2000). Accounting for decision making and control. Irwin, IL: McGraw-Hill.

Recebido em: 31/08/2017

Aprovado em: 01/06/2018

\section{CONTRIBUIÇÕES}

Rogério João Lunkes: Definição do problema de pesquisa e dos objetivos, desenvolvimento da proposição teórica, escolha dos procedimentos metodológicos, análise de dados, elaboração de tabelas, gráficos e figuras, realização de cálculos e projeções, e redação do manuscrito.

Carla Helen Costa: Definição do problema de pesquisa e dos objetivos, desenvolvimento da proposição teórica, realização da revisão bibliográfica e da fundamentação teórica, escolha dos procedimentos metodológicos, coleta de dados, análise de dados, elaboração de tabelas, gráficos e figuras, realização de cálculos e projeções, e redação do manuscrito.

Daiane Antonini Bortoluzzi: Definição do problema de pesquisa e dos objetivos, desenvolvimento da proposição teórica, escolha dos procedimentos metodológicos, análise de dados, elaboração de tabelas, gráficos e figuras, realização de cálculos e projeções, e redação do manuscrito.

Fabricia Silva da Rosa: Definição do problema de pesquisa e dos objetivos, realização da revisão bibliográfica e da fundamentação teórica, escolha dos procedimentos metodológicos, análise de dados, elaboração de tabelas, gráficos e figuras, realização de cálculos e projeções, revisão crítica do manuscrito, redação do manuscrito e adequação do manuscrito às normas da RTA. 